\title{
In Vitro Proliferative Responses and Antibody Titers Specific to Human Acetylcholine Receptor Synthetic Peptides in Patients with Myasthenia Gravis and Relation to HLA Class II Genes
}

\author{
Stefan Brocke," Chaim Brautbar, Lawrence Steinman," Oded Abramsky," Jonathan Rothbard,' Drorit Neumann," \\ Sara Fuchs, * and Edna Mozes* \\ ${ }^{*}$ Department of Chemical Immunology, The Weizmann Institute of Science, Rehovot 76100, Israel; ${ }^{\ddagger}$ Tissue Typing Unit, Department \\ of Clinical Microbiology and Lautenberg Center for General and Tumor Immunology, The Hebrew University, Hadassah Medical \\ Center, Jerusalem 91120, Israel; \$Departments of Pediatrics, Neurology and Genetics, Stanford University School of Medicine, \\ Stanford, CA 94305; "Neuroimmunology Unit, The Hebrew University, Hadassah Medical Center, Jerusalem 91120, Israel; and \\ "Imperial Cancer Research Fund, Lincoln's Inn Fields, London WC2A 3PX, UK
}

\begin{abstract}
To investigate which parts of the acetylcholine receptor are involved in the initiation and development of myasthenia gravis (MG), peptides representing different sequences of the human acetylcholine receptor $\alpha$-subunit were synthesized. These peptides were tested for their ability to stimulate $T$ cells of myasthenic patients and healthy control patients in proliferation assays and to bind to sera antibodies. Three of eight peptides discriminated significantly between the two groups in the proliferation assay, as well as in their ability to bind to serum antibodies. HLA-DR3 and DR5 were associated with proliferative responses to specific $A C h R$ peptides in the group of myasthenics. Acetylcholine receptor epitopes that might play a specific role in myasthenia gravis thus were demonstrated.
\end{abstract}

\section{Introduction}

Myasthenia gravis (MG) ${ }^{1}$ is a very well-characterized autoimmune disorder (1). The symptoms of $\mathrm{MG}$ are caused by an antibody-mediated autoimmune response to the nicotinic acetylcholine receptor (AChR). However, what initiates and regulates the autoimmune reactivity in $\mathrm{MG}$ is still unclear and there is no definitive cure for this disease (1). The higher frequency of certain histocompatibility antigens (HLA-B8, DR3) (2) suggests that the MHC might be involved in regulation of susceptibility to MG. Further, only limited information is available on the $T$ and $B$ cell response to specific epitopes of AChR.

To explain the role of regulatory $T$ cells in the pathogenesis of $\mathrm{MG}$, it is important to study $\mathrm{MG}$ patients for their $\mathrm{T}$ cell immune responses to the relevant antigen, namely human AChR. However, because of minute amounts of $\mathrm{AChR}$ in

Address reprint requests to Dr. Edna Mozes, Department of Chemical Immunology, The Weizmann Institute of Science, Rehovot 76100, Israel.

Received for publication 7 December 1987 and in revised form 29 July 1988.

1. Abbreviations used in this paper: $\mathrm{AChR}$, acetycholine receptor; MG, myasthenia gravis.

J. Clin. Invest.

(C) The American Society for Clinical Investigation, Inc. 0021-9738/88/12/1894/07 \$2.00

Volume 82, December 1988, 1894-1900 human tissue and complicated purification methodologies, it is not possible to obtain AChR in sufficient quantities to enable screening a large number of myasthenic patients. Therefore, very few studies have been performed to measure $T$ cell reactivity of $M G$ patients to the human $A C h R(3,4)$. It appears that the use of synthetic peptides from various regions in the human AChR molecule should alleviate the above problem. Further, this approach will allow for the characterization of a determinant(s) in the human AChR that is recognized by $\mathrm{T}$ cells of MG patients. As $\mathrm{T}$ cell epitopes are recognized in conjunction with class II major histocompatibility products, it might be possible to correlate certain AChR determinants with specific HLA-D products.

In this study, PBL of patients with MG and of healthy control patients were tested for their capacity to proliferate when stimulated in vitro with synthetic peptides that represent different sequences of the human AChR $\alpha$-subunit. All patients were typed for their HLA class I and class II antigens to find out a possible correlation between the proliferative responses and a specific HLA gene(s). Antibody reactivity to the different sequences of the human AChR was also determined in the sera of the patients.

\section{Methods}

MG patients and healthy control patients. 45 patients with MG, (17 males, 28 females) examined at the Department of Neurology, Hadassah Medical Center, Jerusalem, participated in this study after giving informed consent. The patients' age ranged from 18 to $76 \mathrm{yr}$ and duration of disease ranged from 1 to $30 \mathrm{yr}$. The diagnosis was based on clinical manifestations of fluctuating skeletal muscle weakness and fatigability. Serum antibody to AChR (5) was found in 40 patients (89\%). In the five patients without antibody, the diagnosis was confirmed by electrodiagnostic (decremental muscle potential response with a low-frequency stimulation) and pharmacologic (positive testing with edrophonium) criteria. 14 patients underwent thymectomy; thymoma was found in 4.34 patients were treated with prednisone and/or azathioprine. A control group of $\mathbf{3 3}$ healthy donors matched with the patients in sex, age, and ethnic origin was tested simultaneously.

Antigens. Seven peptides representing different regions of the human AChR $\alpha$-subunit and one peptide with a sequence of the Torpedo AChR $\alpha$-subunit were synthesized in our own laboratories. The sequences of the different peptides are shown in Table I. Peptide synthesis and characterization were carried out as previously described $(6,7)$.

$R I A$. Antibody levels specific to the different peptides in the sera of patients and healthy control individuals were determined by solid phase RIA (8). Plates were coated with $100 \mu \mathrm{l}$ of $50 \mu \mathrm{g} / \mathrm{ml} \mathrm{AChR}$ peptides in PBS containing $0.2 \%$ glutaraldehyde and washed with PBS 
Table I. Synthetic Peptides of the AChR $\alpha$-Subunit

\begin{tabular}{|c|c|c|c|c|c|c|c|c|c|c|c|c|c|c|c|c|c|c|c|}
\hline \multirow{2}{*}{$\begin{array}{l}\text { Peptides } \\
\text { p195-212 }\end{array}$} & \multirow{2}{*}{$\begin{array}{c}\text { Species } \\
\text { Human }\end{array}$} & \multicolumn{18}{|c|}{ Sequence } \\
\hline & & Asp & Thr & Pro & Tyr & Leu & Asp & Ile & Ile & Tyr & His & Phe & Val & Met & Gln & Arg & Leu & Pro & Leu \\
\hline p257-269 & Human & Leu & Leu & Val & Ile & Val & Glu & Leu & Ile & Pro & Ser & Thr & Ser & Ser & & & & & \\
\hline p310-327 & Human & Asn & Trp & Val & Arg & Lys & Ile & Phe & Ile & Asp & Thr & Ile & Pro & Asn & Ile & Met & Phe & Phe & Ser \\
\hline p169-181 & Human & Asn & Phe & Met & Glu & Ser & Gly & Glu & Trp & Val & Ile & Lys & Glu & Ser & & & & & \\
\hline p183-196 & Human & Gly & Trp & Lys & His & Ser & Val & Thr & Tyr & Ser & Cys & Cys & Pro & Asp & Thr & & & & \\
\hline p185-196 & Human & Lys & His & Ser & Val & Thr & Tyr & Ser & Cys & Cys & Pro & Asp & Thr & & & & & & \\
\hline p351-368 & Human & Ile & Ser & Gly & Lys & Pro & Gly & Pro & Pro & Pro & Met & Gly & Phe & $\mathrm{His}$ & Ser & Pro & Leu & Ile & Lys \\
\hline p394-409 & Torpedo & Asn & Ala & Ala & Glu & Glu & Trp & Lys & Tyr & Val & Ala & Met & Val & Ile & Asn & His & Ile & & \\
\hline
\end{tabular}

containing $0.2 \%$ gelatin (E. Merck, Darmstadt, FRG), and $50 \mu$ lof the sera to be assayed was added in different dilutions for $4 \mathrm{~h}$ incubation. ${ }^{125}$ I-labeled protein A (Sigma Chemical Co., St. Louis, MO) $\left(10^{5} \mathrm{cpm} /\right.$ well) was then added and radioactivity of each well was measured, after extensive washes, in a gamma counter (Packard). Results are expressed as mean counts per minute of duplicates. Sera were considered positive if counts per minute were above mean counts per minute of controls +2 SD.

Proliferative responses. PBL of myasthenic and healthy donors were isolated from the whole blood by Ficoll-Hypaque density gradient centrifugation (Pharmacia Fine Chemicals, Uppsala, Sweden). The assays were performed in flat-bottom microtiter plates (Nunc, Roskilde, Denmark) at a cell concentration of $2 \times 10^{5} /$ well in enriched RPMI medium supplemented with $10 \%$ autologous serum (9). Different concentrations of the peptides representing sequences of the human AChR $\alpha$-subunit were added to the wells. Phytohemaglutinin M (PHA; Difco Laboratories, Detroit, MI) and Con A (Bio-Yeda, Nes Ziona, Israel) ( $2 \mu \mathrm{g} /$ well) were used as controls for culture conditions. Cultures were incubated for $6 \mathrm{~d}$. For the last $16 \mathrm{~h},\left[{ }^{3} \mathrm{H}\right]$ thymidine $(0.5 \mu \mathrm{Ci}$ of $5 \mathrm{Ci} / \mathrm{mmol}$, Nuclear Research Center, Negev, Israel) was added to the cultures. Thereafter, cells were harvested and radioactivity was counted. Results are expressed as mean counts per minute of triplicate cultures $\pm \mathrm{SD}$ or as stimulation indices (the ratio of mean counts per minute at the optimal peptide concentration to mean counts per minute without antigen). A stimulation index $>3$ was considered positive.

HLA examination. PBL were isolated from heparinized blood samples of donors as described above. HLA-A,B,C antigens were determined by the microcytotoxity technique (10). HLA-DR,DQ typing was performed on T cell-depleted, B cell-enriched lymphocytes by extended incubation cytotoxicity testing (11). Each individual was typed at least twice. Generally, four to six alloantisera were used to test for each specificity. The sera used to determine the HLA-A,B,C, DR and DQ antigens, were of local origin, from other investigators and some were from the Ninth Histocompatibility Workshop "genetic" and "disease" set.

Statistical analyses. Significance of differences between blood samples of patients and controls in proliferative responses and antibody levels was examined using the Chi-square test with Yate's correction or Fisher's exact test. A $P$ value of $<0.05$ was considered to be significant. It should be noted that not all patients that were HLA typed had proliferation and/or antibody tests performed; conversely, not all patients that had these tests performed could be HLA typed.

\section{Results}

Proliferative responses of $P B L$ of $M G$ patients to peptides representing sequences of the human $A C h R$. PBL of MG patients and of healthy controls were tested for their ability to proliferate in the presence of an in vitro stimulus with the various peptides representing regions of the AChR $\alpha$-subunit that are listed in Table I. Representative results of proliferative re- sponses of PBL of one control and three MG patients to one peptide (p195-212) are shown in Fig. 1. As can be seen, the PBL of the healthy control and of one of the patients did not proliferate in the presence of p195-212, whereas significant proliferative responses were observed with PBL of the other two patients, although they differed in the dose of peptide required for maximum proliferation.

The stimulation indices of the proliferative responses of the 28 patients and 33 controls tested with p195-212 are presented in Fig. 2. A stimulation index $>3$ was considered positive. It is clear from the figure that a significantly larger number of patients responded to p195-212 compared with the healthy controls $(P<0.001)$.

Table II summarizes the results of the proliferative assays specific to eight sequences of the AChR performed with PBL of MG patients and healthy controls. As shown in the table, the myasthenic patients responded better to all the peptides. However, only three peptides (p195-212, p257-269, and p310-327) could discriminate significantly between MG patients and controls on the basis of the proliferative responses of PBL. Scatter diagrams for responses to p257-269 and p310-327 are shown in Figs. 3 and 4. The differences between MG patients and controls were the most significant when the responses to p195-212 were measured (Table II).

Antibody levels specific to synthetic peptides representing $A C h R$ in sera of $M G$ patients. It was of interest whether sera of patients with myasthenia gravis possess antibodies that react with the different peptides representing the AChR $\alpha$-subunit.

Sera of MG patients and of controls were tested by RIA to detect antibody titers specific to seven peptides used in this study. Fig. 5 demonstrates antibody levels in sera of all MG patients and healthy controls tested with p195-212, expressed in counts per minute. It is clear from the figure that the vast

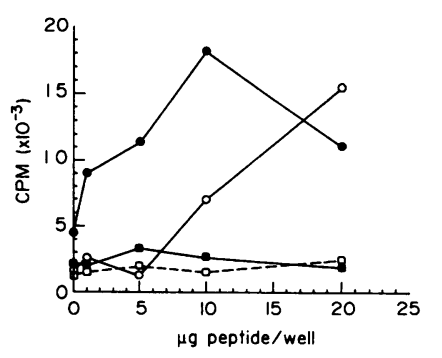

Figure 1. Proliferative responses to p195-212. $2 \times 10^{5} /$ well (PBL) of three MG patients (-) and one healthy control (---) were cultured with different doses of p195-212 as indicated in the figure. After $6 \mathrm{~d}$ in culture, $\left[{ }^{3} \mathrm{H}\right]$ thymidine $(0.5 \mu \mathrm{Ci})$ was added. $16 \mathrm{~h}$ later, cells were harvested and radioactivity was counted. Results are expressed as mean counts per minute of triplicate cultures. 


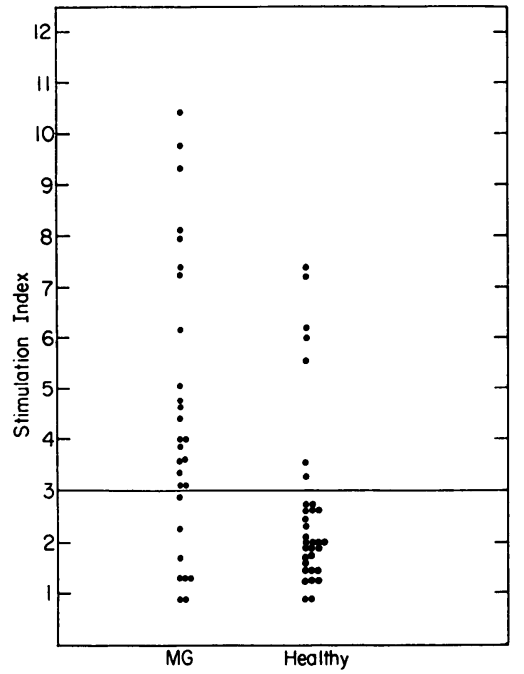

Figure 2. Proliferative responses of PBL of MG patients and healthy controls to p195-212. PBL of 28 MG patients and 33 healthy controls were cultured with different concentrations of p195-212 as indicated in Fig. 1. Results are expressed as stimulation indices.

majority (31 of 39) of sera of the MG patients possessed antibodies that reacted with p195-212, whereas sera of only 2 of the 31 healthy controls tested reacted with this peptide ( $P$ $<0.001)$. Thus, p195-212 also discriminated significantly between patients and controls based on sera antibody titers.

Table III summarizes the percentages of positive antibody titers specific to the seven peptides in sera of MG patients and controls. It can be seen in the table that the percentages of sera with antibody titers specific to p195-212, p257-269, and p310-327 are significantly higher than those determined in sera of controls. Scatter diagrams for antibody responses to p257-269 and p310-327 are shown in Figs. 6 and 7. As shown for the proliferative responses, the differences obtained with p195-212 were the most significant. No significant differences could be detected between MG patients and controls when antibody titers were measured to p169-181, p185-196, p351-368, and p394-409. In both groups the number of positive sera to the latter peptides was found to be small.

$H L A$ typing of $M G$ patients. The HLA-A,B,C, DR, and DQ phenotypes of $45 \mathrm{MG}$ patients were tested. 15 patients (33\%) possessed HLA-B8. 14 of the 45 patients $(31 \%)$ possessed HLA-DR3. In 12 cases of the latter, DR3 occurred jointly with HLA-B8. Note that both HLA-B8 and DR3 are present in relatively low frequencies of 9.4 and $10.4 \%$, respectively, in the Israeli population. Moreover, the joint occurrence of B8 and DR3 in the healthy Israeli population is rather low $(5.7 \%)$ but

Table II. Percentages of Positive Proliferative PBL Responses of MG Patients and Healthy Controls to Peptides Representing Different Sequences of the Human AChR

\begin{tabular}{lll}
\hline Peptides & \multicolumn{1}{c}{ MG patients } & \multicolumn{1}{c}{ Healthy controls } \\
\hline p195-212 & $72.5 \%(20 / 28)$ & $21.2 \%(7 / 33) P<0.001$ \\
p257-269 & $56 \%(14 / 25)$ & $16 \%(4 / 25) P<0.01$ \\
p310-327 & $44.4 \%(12 / 27)$ & $15.6 \%(5 / 32) P<0.01$ \\
p169-181 & $26.9 \%(7 / 26)$ & $9.5 \%(2 / 21)$ \\
p183-196 & $26.9 \%(7 / 26)$ & $19.2 \%(5 / 26)$ \\
p185-196 & $15.4 \%(4 / 26)$ & $3.8 \%(1 / 26)$ \\
p351-368 & $35.7 \%(10 / 28)$ & $26.9 \%(7 / 26)$ \\
p394-409 & $26.9 \%(7 / 26)$ & $19.2 \%(5 / 26)$ \\
\hline
\end{tabular}

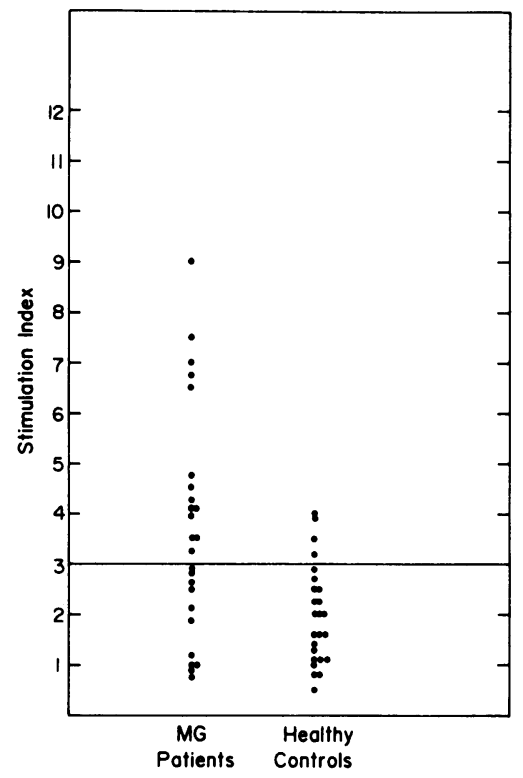

Figure 3. Proliferative responses of PBL of MG patients and healthy controls to p257-269. PBL of 25 MG patients and 25 healthy controls were cultured with different concentrations of p257-269 as indicated in Fig. 1. Results are expressed as stimulation indices. significantly higher $\left(\chi^{2}=11.3, P<0.001\right)$ in the Israeli MG patients. The relative risk for B8, DR3 carriers is 6.1 .

It was tested whether a possible correlation exists between the proliferative capacity of PBL of MG patients and control individuals to the various peptides of the AChR and HLA. Table IV displays the class I and class II HLA types and the proliferation and antibody responses to peptides p195-212, p257-269, and p310-327. Table $V$ demonstrates that all seven HLA-DR3 type patients responded to p257-269 $(P<0.0003)$. The one HLA-DR3 healthy individual also responded to p257-269. Non-HLA-DR3 patients also responded to p257-269, but less frequently ( 7 of 7 vs. 6 of $16, P<0.006$ ). Table VI demonstrates that 10 of $12(83 \%)$ of the HLA-DR5 myasthenic patients responded to $\mathrm{p} 195-212$ by proliferation compared with 2 of 12 (17\%) of myasthenic DR5 carriers who did not respond. In the group of HLA-DR5 healthy controls, the distribution was different, with 3 of $14(21 \%)$ responding and 11 of 14 not responding to p195-212 $(P<0.007)$. Non-

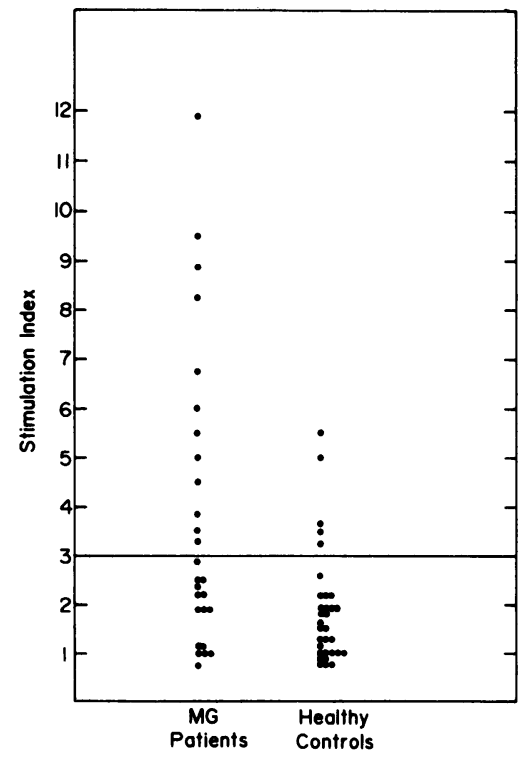

Figure 4. Proliferative responses of PBL of MG patients and healthy controls to p310-327. PBL of 27 MG patients and 32 healthy controls were cultured with different concentrations of p310-327 as indicated in Fig. 1. Results are expressed as stimulation indices. 


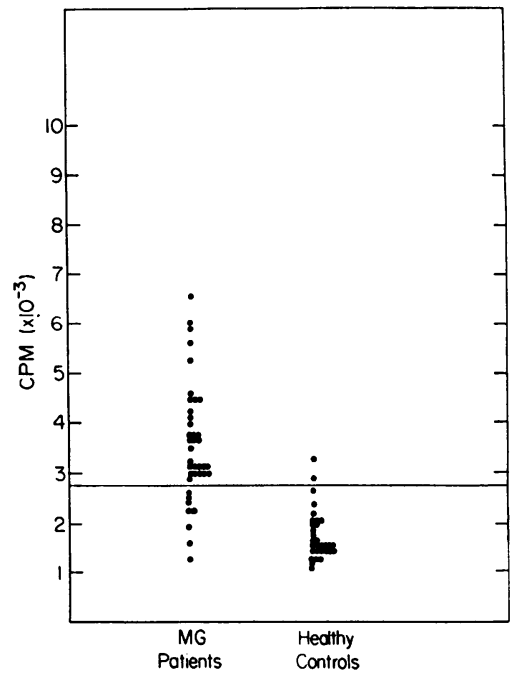

Figure 5. Serum antibody levels of MG patients and healthy controls to p195-212. Sera of 39 MG patients and 31 healthy controls were tested for their antibody titers against p195-212 in a solidphase RIA. Results are expressed as mean counts per minute of duplicates.
HLA-DR5 myasthenics also responded frequently to p195-212 (9 of 15 vs. 10 of $12, P=$ NS). No correlation could be observed between proliferative responses to $p 310-327$ and any HLA-DR antigen. Antibody reactivity to p195-212, p257-269, and p310-327 in the sera of MG patients was not associated with any particular HLA type.

\section{Discussion}

In this report, we have demonstrated cellular and antibody responses to synthetic peptides that represent sequences of the human acetylcholine receptor in patients with MG. Furthermore, the proliferative responses to two of the peptides appear to correlate with specific HLA-DR determinants.

To explain the role of regulatory $T$ cells in the pathogenesis of $M G$, it is critical to investigate $T$ cell responses of $M G$ patients to antigens relevant for the disease. Synthetic peptides from various regions in the AChR molecule have been used for structure and function analyses of the AChR and of MG (6, $12-15$ ). In this report, seven peptides that represent sequences of the human AChR $\alpha$-subunit and one peptide with a region of the Torpedo AChR (Table I) were used to screen T cell proliferative responses of MG patients. Three of the peptides (Table II and Figs. 2-4) discriminated significantly between MG patients and healthy controls. These results suggest certain sequences in the AChR molecule that might be critical in

Table III. Percentages of Positive Serum Antibody Levels of MG Patients and Healthy Controls to Peptides Representing Different Sequences of the Human AChR

\begin{tabular}{|c|c|c|}
\hline Peptides & MG patients & Healthy controls \\
\hline p195-212 & $79.5 \%(31 / 39)$ & $6.5 \%(2 / 31) P<0.0001$ \\
\hline p257-269 & $47.5 \%(19 / 40)$ & $3.3 \%(1 / 30) P<0.001$ \\
\hline p310-327 & $49 \% \quad(21 / 43)$ & $6.5 \%(2 / 31) P<0.001$ \\
\hline p169-181 & $14 \% \quad(6 / 43)$ & $6.5 \%(2 / 31)$ \\
\hline p185-196 & $5 \% \quad(2 / 40)$ & $3.3 \%(1 / 30)$ \\
\hline p351-368 & $5 \% \quad(2 / 40)$ & $3.3 \%(1 / 30)$ \\
\hline p394-409 & $16 \% \quad(7 / 43)$ & $6.5 \%(2 / 31)$ \\
\hline
\end{tabular}

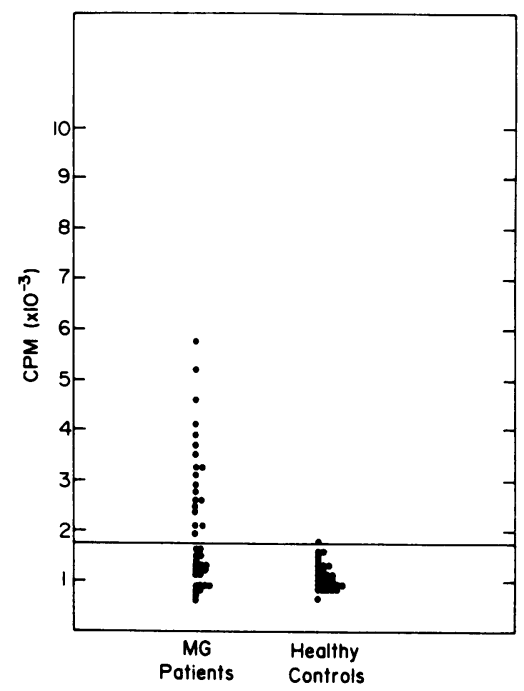

Figure 6. Serum antibody levels of MG patients and healthy controls to p257-269. Sera of $40 \mathrm{MG}$ patients and 30 healthy controls were tested for their antibody titers against p257-269 in a solidphase RIA. Results are expressed as mean counts per minute of duplicates. the pathogenesis of MG. It should be noted that p195-212, p257-269, and p310-327 were chosen to conform to a pattern present in many $T$ cell epitopes $(16,17)$. The pattern consists of peptides containing a sequence composed of a charged residue or glycine, followed by two hydrophobic residues. The fourth residue is charged or polar. If the fourth residue was hydrophobic, the next position has a polar residue $(16,17)$. Peptides p169-181, p183-196, p185-196, p351-362, and p394-409 were not as immunogenic as the three peptides chosen by the predictive template for $T$ cell epitopes $(16,17)$. Other studies (14) have shown that PBL of myasthenic patients proliferated significantly in the presence of p169-181 and p351-368. Using various antibodies, the latter peptide was demonstrated to reside in a highly immunogenic region of the AChR (18).

The detection of antibodies to AChR receptors in the serum of MG patients provides an approach to diagnosing and monitoring MG patients $(19,20)$. Therefore, it is of interest that peptides p195-212, p257-269, and p310-327 discriminated significantly between MG patients and controls based on sera antibodies that bound to these peptides (Table III and Figs. 5-7). In agreement with the proliferative assays, p195-212 was the most discriminative with $79.5 \%$ of the pa-

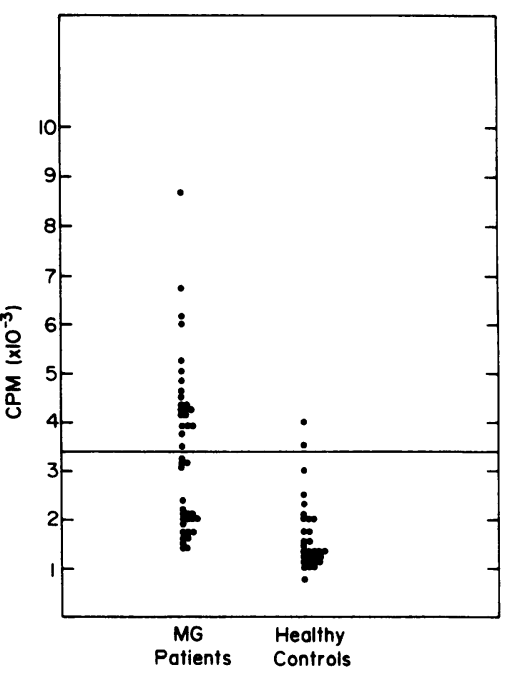

Figure 7. Serum antibody levels of MG patients and healthy controls to $\mathrm{p} 310-327$. Sera of $43 \mathrm{MG}$ patients and 31 healthy controls were tested for their antibody titers against phase RIA. Results are expressed as mean counts per minute of duplicates. p310-327 in a solid- 


\begin{tabular}{|c|c|c|c|c|c|c|c|c|c|c|c|}
\hline \multirow[b]{2}{*}{ Patients } & \multirow[b]{2}{*}{ A } & \multirow[b]{2}{*}{$\mathrm{Cw}$} & \multirow[b]{2}{*}{ B } & \multirow[b]{2}{*}{ DR } & \multirow[b]{2}{*}{ DQw } & \multicolumn{3}{|c|}{ Proliferation to peptides } & \multicolumn{3}{|c|}{ Antibodies to peptides } \\
\hline & & & & & & p195-212 & p257-269 & p310-327 & p195-212 & p257-269 & p310-327 \\
\hline K.B. & 30,28 & 6,8 & 13,14 & 7,1 & 2,1 & ND & ND & ND & ND & + & - \\
\hline T.E. & 1,24 & 4,7 & 8,35 & $4,-$ & $3,-$ & ND & ND & ND & ND & + & + \\
\hline M.N. & 1,11 &,-- & $51,-$ & 2,5 & 1,3 & ND & ND & ND & ND & + & + \\
\hline S.E. & 26,30 &,-- & $38,-$ & $4,-$ & $3,-$ & ND & ND & ND & ND & - & + \\
\hline T.I. & 23,26 & $4,-$ & 8,38 & $3,-$ & $2,-$ & ND & ND & ND & + & - & - \\
\hline M.E. & 28,26 & $3,-$ & w55, 38 & 2,4 & 1,3 & ND & ND & ND & + & - & - \\
\hline B.B. & 2,24 & $7,-$ & $38, w 41$ & $5, w 6$ & 3,1 & ND & ND & ND & + & - & - \\
\hline S.S. & $2,-$ & 3,4 & 35, w60 & $2, w 10$ & 1,1 & ND & ND & ND & + & - & - \\
\hline Z.G. & 1,26 & $7,-$ & 8, w63 & $3, w 6$ & 1,2 & ND & ND & ND & + & - & + \\
\hline Sa.E. & 2,26 & $7,-$ & 8,38 & $3,-$ & $2,-$ & ND & ND & ND & + & - & + \\
\hline T.J. & $2,-$ & 5,4 & 35,44 & ND & ND & ND & ND & ND & + & - & + \\
\hline H.M. & $2,-$ & 4,7 & 35,8 & 5,3 & 3,2 & ND & ND & ND & + & - & + \\
\hline K.S. & 28,29 &,- 8 &,- 14 & 1,5 & 1,3 & ND & ND & ND & + & - & + \\
\hline M.H. & 1,24 & $4,-$ & 35,38 & ND & ND & ND & ND & ND & + & - & + \\
\hline G.T. & 30,28 & 6,7 & w57, w41 & $5,-$ & $3,-$ & - & - & - & - & ND & + \\
\hline S.M. & 11,29 &,-- & 51,35 & w6, - & $1,-$ & + & + & - & + & ND & - \\
\hline C.E. & 2,26 &,-- & $38, w 41$ & $4,-$ & $3,-$ & - & - & - & + & - & - \\
\hline D.H. & $1,-$ & $7,-$ & 8,7 & 3 , w6 & 1,2 & ND & ND & ND & - & ND & + \\
\hline B.D. & 3,28 & $8,-$ & 14, w61 & $1,-$ & $1,-$ & - & ND & ND & + & - & - \\
\hline Sh.S. & 23,26 &,-- & w41, 38 & 4,5 & 3,3 & + & ND & - & + & - & - \\
\hline D.A. & 1,29 &,-- & 7,51 & $5,-$ & $3,-$ & + & + & + & - & + & - \\
\hline F.S. & 23,32 & $4,-$ & 44,27 & 4,5 & 3,3 & + & - & + & - & + & - \\
\hline S.A. & $24,-$ & $4,-$ & $35,-$ & ND & ND & ND & - & + & + & + & - \\
\hline Su.A. & 2,31 &,-- & 8,38 & 3,4 & 2,3 & + & + & + & + & + & - \\
\hline S.R. & 3,24 & 8,7 & 8,14 & 3,1 & 2,1 & + & + & - & + & - & - \\
\hline B.N. & $26,-$ & $8,-$ & 14, w57 & ND & ND & + & + & + & + & + & + \\
\hline N.T. & 24,30 &,-- & $18, w 41$ & 5,7 & 3,2 & + & - & ND & + & + & - \\
\hline Sh.M. & 2,28 & $2,-$ & 27,44 & $2, w 8$ & $1,-$ & - & - & - & - & - & - \\
\hline S.K. & 23,30 & 4,7 & 44,13 & 5,7 & 3,2 & + & - & - & ND & ND & ND \\
\hline G.S. & $2,-$ &,-- & $8,-$ & $3,-$ & $2,-$ & + & + & + & + & + & + \\
\hline N.B. & 24,32 & $2,-$ & 35, w62 & $5,-$ & $3,-$ & - & - & - & + & + & + \\
\hline K.D. & 1,31 & 4,7 & 8,35 & 3,4 & 2,3 & + & + & - & + & - & - \\
\hline Z.R. & 2,11 & $2,-$ & w52, - & $3,-$ & $2,-$ & + & + & + & + & + & + \\
\hline K.I. & 11,2 & 6,7 & $8, w 50$ & 3, w6 (w13) & 2,1 & + & + & + & + & + & + \\
\hline L.H. & 1,2 & 6,7 & 7, w57 & 7,1 & 2,1 & - & - & - & + & - & - \\
\hline H.H. & 3,26 & 4,7 & 35,49 & 2,5 & 1,3 & + & + & + & + & + & + \\
\hline H.S. & 3,24 & $4,-$ & 35, w60 & 3, w6 (w13) & 2,1 & + & + & + & + & + & + \\
\hline B.P. & 3,24 & $4,-$ & $35, w 41$ & 4,5 & 3,3 & + & - & - & + & - & - \\
\hline R.M. & 3,24 & 4,7 & 8,35 & 3,2 & 2,1 & - & ND & - & + & + & + \\
\hline P.M. & 1,24 & 4,7 & 8,35 & $5,-$ & $3,-$ & + & + & + & + & - & - \\
\hline S.N. & 1,24 & 6,1 & w57, 51 & w6 (w14), 5 & 1,3 & + & + & + & + & + & + \\
\hline S.U. & 1,26 & $7,-$ & 8,18 & 3,4 & 2,3 & - & ND & - & - & + & + \\
\hline G.C. & 2,3 & $7,-$ & 44, w52 & 5,2 & 3,1 & + & + & - & - & + & + \\
\hline T.R. & 1, w33 & 7,8 & 14,8 & 1,4 & 1,3 & + & - & - & - & - & - \\
\hline
\end{tabular}

+ and - indicate positive and negative responses (see Methods).

tients' sera reacting with it, in contrast to $6.5 \%$ of the sera taken from controls (Fig. 4, Table III), thus strengthening the evidence for a role that this sequence might have in the pathogenesis of the disease.

The majority of patients responded to more than one peptide in both the proliferative and antibody assays (Table IV). These data are important when considering the implications for therapy. Thus, the response to more than a single epitope will need to be suppressed.
The significance of a certain frequency of positive proliferative responses in healthy controls is unclear. The significance of the proliferation results is bolstered by the detection of a large percentage (48-80\%) of MG sera that bind p195-212, p257-269, and p310-327. Again, the meaning of peptide binding in few sera of normal individuals (3-7\% of healthy controls) is unresolved. A potential explanation is a possible cross-reaction between sequences of the AChR $\alpha$-subunit and certain bacteria and viruses. Indeed, shared antigenic determi- 
Table V. Correlation between HLA-DR3 and Proliferative Responses to p257-269

\begin{tabular}{llc}
\hline & \multicolumn{1}{c}{ Responder } & Nonresponder \\
\hline HLA-DR3 myasthenic & $7 / 7^{* \ddagger}(100 \%)$ & $0 / 7^{*}(0 \%)$ \\
Not HLA-DR3 myasthenic & $6 / 16^{\ddagger}(38 \%)$ & $10 / 16(62 \%)$ \\
HLA-DR3 healthy & $1 / 1(100 \%)$ & $0 / 0(0 \%)$ \\
Not HLA-DR3 healthy & $3 / 17(18 \%)$ & $14 / 17(82 \%)$
\end{tabular}

* $P<0.0003$, Fisher's exact test.

${ }^{\ddagger} P<0.006$, Fisher's exact test.

nants between the AChR $\alpha$-subunit and membrane proteins of several bacteria have been reported (21).

A higher incidence of HLA-B8, DR3 has been reported for MG patients (2). Indeed, of the Israeli group of MG patients we tested, $27 \%$ were determined to express jointly HLA-B8, DR3, a significantly higher percentage from that observed in the Israeli healthy control group. This observation is of special interest because many autoimmune diseases such as multiple sclerosis, systemic lupus erythematosus, and rheumatoid arthritis in the Israeli population do not follow the reported HLA associations (22-24). Of more interest are the correlations found between the proliferative capacities to p195-212 and p257-269 and specific HLA-DR determinants. A high percentage of the responders to $\mathrm{p} 195-212$ possess HLA-DR5, whereas all HLA-DR3 individuals tested responded to p257-269 (Tables V and VI). Note that MG patients that are not HLADR3 also responded to p257-269 (6 of 16, Table V) and nonHLA-DR5 patients responded to p195-212 (9 of 15, Table VI). MG is linked to class II major histocompatibility genes in man and in its experimental counterpart, experimental autoimmune myasthenia gravis, in mice (25-27). Class II HLA polymorphisms have been described in MG patients who are HLA-DR 3 (25). The HLA-DR $\alpha$ and DR $\beta$ genes and HLADQ $\alpha$ and $\beta$ genes have been sequenced in Caucasoid MG patients. No sequence difference was seen between MG patients and healthy controls (28). Thus, polymorphism, linked to $M G$ in the HLA-D region may map to HLA-DP or perhaps $\mathrm{DX}$ and DZ. In the mouse, responsiveness to AChR is controlled by the I-A $\beta$ chain $(26,27)$.

This study suggests that peptides of AChR are particularly immunogenic for certain HLA-DR types. HLA-DR5 individuals responded better than healthy controls to p195-212. Moreover, all HLA-DR3 respond to p257-269. It has been demonstrated that immunogenic peptides bind directly to class II MHC products (29). In this context, it is possible that p195-212 binds more readily with an MG-associated class II

Table VI. Correlation between HLA-DR5 and Proliferative Responses to p195-212

\begin{tabular}{lcr}
\hline & Responder & Nonresponder \\
\hline HLA-DR5 myasthenic & $10 / 12^{*}(83 \%)$ & $2 / 12(17 \%)$ \\
Not HLA-DR5 myasthenic & $9 / 15(60 \%)$ & $6 / 15(40 \%)$ \\
HLA-DR5 healthy & $3 / 14^{*}(21 \%)$ & $11 / 14(79 \%)$ \\
Not HLA-DR5 healthy & $3 / 15(20 \%)$ & $12 / 15(80 \%)$
\end{tabular}

$* \chi^{2}=7.6, P<0.01$.
HLA product, whereas p257-269 binds preferentially to HLADR3. The fact that HLA-DR3 class II molecules have identical sequences in MG patients and controls may underlie the observation that some healthy individuals respond to some AChR peptides (28). Further, various polymorphic residues in the HLA-class II antigen binding cleft are shared by various HLA-DR and DQ types (28). This may account for the fact that individuals with different HLA-class II genotypes can respond to a given $\mathrm{AChR}$ peptide.

These studies represent one of the first attempts to analyze the determinants of the AChR molecule that are involved in the aberrant immune response in MG in humans. Hohlfeld and others have reported responses to three $\mathrm{NH}_{2}$-terminal AChR peptides in $\mathrm{T}$ cell lines from two MG patients that react with Torpedo AChR (30). Atassi and colleagues have studied the response in inbred mouse strains to various $A C h R$ peptides from the $\alpha$ subunit of Torpedo AChR (31). Our studies demonstrate significant differences between MG patients and healthy controls in the immune response potential to various sequences of the human AChR and its association with HLA. Other epitopes of $\mathrm{AChR}$, when tested, may also be shown to play a critical role in the pathogenesis of MG.

\section{Acknowledgments}

The authors are indebted to Dr. T. Brenner, Dr. I. Cohen, Mrs. M. Dayan and Mrs. L. Sherman for their assistance. The discussions with Prof. Newsom-Davis regarding his results with AChR peptides are appreciated.

This research was supported in part by the Los Angeles and California Chapters of the Myasthenia Gravis Foundation.

\section{References}

1. Lindstrom, J. 1985. Immunobiology of myasthenia gravis, experimental autoimmune myasthenia gravis, and Lambert-Eaton syndrome. Annu. Rev. Immunol. 3:109-131.

2. Compston, D., A. Vincent, J. Newsom-Davis, and J. Batchelor. 1981. Clinical, pathological, HLA antigen and immunological evidence for disease heterogeneity in myasthenia gravis. Brain. 103:579601 .

3. McQuillen, D. P., S. M. Koethe, and M. P. McQuillen. 1983. Cellular response to human acetylcholine receptor in patients with myasthenia gravis. J. Neuroimmunol. 5:59-65.

4. Hohlfeld, R., K. V. Toyka, K. Heininger, H. Grosse-Wilde, and I. Kalies. 1984. Autoimmune human T lymphocytes specific for acetylcholine receptor. Nature (Lond.). 310:244-246.

5. Abramsky, O., R. P. Lisak, T. Brenner, A. Zeidman, and Y. Beyth. 1979. Significance in neonatal myasthenia gravis of inhibitory effect of amniotic fluid on binding of antibodies of acetylcholine receptor. Lancet. ii:1333-1335.

6. Neumann, D., J. M. Gershoni, M. Fridkin, and S. Fuchs. 1985. Antibodies to synthetic peptides as probes for the binding site on the $\alpha$-subunit of the acetylcholine receptor. Proc. Natl. Acad. Sci. USA. 82:3490-3493.

7. Zamvil, S., D. Mitchell, A. Moore, K. Kitamura, L. Steinman, and J. Rothbard. 1986. T-cell epitope of the autoantigen myelin basic protein that induces encephalomyelitis. Nature (Lond.). 324:258-260.

8. Axelrod, O., and E. Mozes. 1986. Analysis of the biological functions and fine specificity of $(T, G)-A-L$ specific $T$ cell clones. Immunobiology. 172:99-109.

9. Katz, D., Z. Bentwich, N. Eshhar, I. Löwy, and E. Mozes. 1981. Immune response potential to poly (Tyr,Glu)-poly(DLAla)-poly (Lys) of human T cells of different donors. Proc. Natl. Acad. Sci. USA. 78:4505-4509. 
10. Mittal, K. K., M. R. Mickey, D. P. Singal, and P. I. Terasaki. 1968. Refinement of microdroplet lymphocyte cytotoxicity test. Transplantation. 6:913-927.

11. Terasaki, P. I., D. Bernoco, M. S. Park, G. Ozturk, and Y. Iwaki. 1978. Microdroplet testing for HLA-A,B,C and D antigens. Am. J. Clin. Pathol. 69:103-120.

12. Neumann, D., D. Barchan, A. Safran, J. M. Gershoni, and S. Fuchs. 1986. Mapping of the $\alpha$-bungarotoxin binding site within the $\alpha$-subunit of the acetylcholine receptor. Proc. Natl. Acad. Sci. USA. 83:3008-3011.

13. Wilson, P. T., T. L. Lentz, and E. Howrot. 1985. Determination of the primary amino acid sequence specifying the $\alpha$-bungarotoxin binding site on the $\alpha$-subunit of the acetylcholine receptor from Torpedo California. Proc. Natl. Acad. Sci. USA. 82:8790-8794.

14. Berrih-Aknin, S., S. Cohen-Kaminsky, D. Neumann, J. F. Bach, and S. Fuchs. 1987. Proliferative responses to acetylcholine receptor peptides in myasthenia gravis. J. Neuroimmunol. 16:17-22.

15. McCormick, D. J., G. E. Griemann, Z. X. Huang, E. H. Lambert, and V. A. Lennon. 1987. Myasthenogenicity of human acetylcholine receptor synthetic $\alpha$-subunit peptide 125-147 does not require intramolecular disulfide cyclization. J. Immunol. 139:2615-2619.

16. Rothbard, J. B., and W. R. Taylor. 1988. A sequence pattern common to T cell epitopes. EMBO (Eur. Mol. Biol. Organ.) J. 7:93-100.

17. Rothbard, J. B. 1986. Peptides and the cellular immune response. Ann. de Inst. Pasteur (Paris). 137:518-526.

18. Souroujon, M. C., D. Neumann, S. Pizzighella, A. Safran, and S. Fuchs. 1986. Localization of a highly immunogenic region on the acetylcholine receptor $\alpha$-subunit. Biochem. Biophys. Res. Commun. 135:82-89.

19. Lindstrom, J. M., M. E. Seybold, V. A. Lennon, S. Whittingham, and D. Duane. 1976. Antibody to acetylcholine receptor in myasthenia gravis: prevalence, clinical correlates and diagnostic value. Neurology. 26:1054-1059.

20. Limburg, P., T. The, E. Hummel-Tappel, and H. Oosterhuis. 1983. Anti-acetylcholine receptor antibodies in myasthenia gravis. I. Relation to clinical parameters in 250 patients. J. Neurol. Sci. 58:357370.

21. Stefansson, K., M. E. Dieperink, D. P. Richman, C. M. Gomez, and L. S. Martan. 1985. Sharing of antigenic determinants between the nicotinic acetylcholine receptor and proteins in Escherichia coli, Proteus vulgaris and K. Pneumoniae. N. Engl. J. Med. 312:224-225.

22. Shalev, Y., Z. Bentwich, D. Katz, C. Brautbar, and E. Mozes. 1985. (T,G)-A-L specific immune response potential and HLA typing of Israeli patients with systemic lupus erythematosus. Clin. Exp. Immunol. 60:355-367.

23. Brautbar, C., A. Amar, N. Cohen, J. Oksenberg, I. Cohen, E. Kahana, D. Bloch, M. Alter, and H. Crosse-Wilde. 1982. HLA-D typing in multiple sclerosis: Israelis tested with European homozygous typing cells. Tissue Antigens. 19:189-197.

24. Tishler, M., C. Brautbar, S. Battat, M. Dayan, and E. Mozes. 1987. Analysis of the antigen specific helper T cell function and HLADR of Israeli patients with rheumatoid arthritis (RA). Tissue Antigens. 30:229-234.

25. Bell, J., S. Smoot, C. Newby, K. Toyka, L. Rassenti, K. Smith, R. Hohlfeld, H. O. McDevitt, and L. Steinman. 1986. HLA-DQ beta chain polymorphism linked to myasthenia gravis. Lancet. ii:10581060.

26. Christadoss, P., J. Lindstrom, R. Melvold, and N. Talal. 1985. I-A subregion mutation prevents immune EAMG. Immunogenetics. 21:33-39.

27. Waldor, M., S. Sriram, H. O. McDevitt, and L. Steinman. 1983. In vivo therapy with monoclonal anti I-A antibody suppresses immune response to AChR. Proc. Natl. Acad. Sci. USA. 80:2713-2717.

28. Todd, J. A., H., Acha-Orbea, J. I. Bell, N. Chao, Z. Fronek, C. Jacob, M. McDermott, A. Sinha; L. Timmerman, L. Steinman, and H. O. McDevitt. 1988. A molecular basis for major histocompatibility complex class II associated autoimmunity. Science (Wash. DC). 240:1003-1009.

29. Babbitt, B., P. Allen, G. Matsueda, E. Haber, and E. Unanne. 1985. Binding of immunogenic peptides to Ia histocompatibility molecules. Nature (Lond.). 317:359-361.

30. Hohlfeld, R., K. Toyka, L. Miner, S. Walgrave, and B. ContiTronconi. 1988. Amphipathic segment of the nicotinic AChR $\alpha$-subunit contains epitopes recognized by $\mathrm{T}$-lymphocytes in myasthenia gravis. J. Clin. Invest. 81:657-660.

31. Atassi, M. Z., B. Mulac-Jericevic, T. Yokoi, and T. Manshour. 1987. Localization of the functional sites on the $\alpha$ chain of acetylcholine receptor. Fed. Proc. 46:2538-2547. 